\title{
Optimization of Running Blade Prosthetics Utilizing Crow Search Algorithm Assisted by Artificial Neural Networks
}

\author{
Manuel Javier Rosel Solis ${ }^{1}$ - José Omar Dávalos Ramírez ${ }^{2,}{ }^{*}$ - Javier Molina Salazar² - \\ Juan Antonio Ruiz Ochoa ${ }^{1}$ - Antonio Gómez Roa ${ }^{1}$ \\ ${ }^{1}$ Autonomous University of Baja California, México \\ ${ }^{2}$ Autonomous University of Ciudad Juárez, México
}

A crow search algorithm (CSA) was applied to perform the optimization of a running blade prosthetics (RBP) made of composite materials like carbon fibre layers and cores of acrylonitrile butadiene styrene (ABS). Optimization aims to increase the RBP displacement limited by the Tsai-Wu failure criterion. Both displacement and the Tsai-Wu criterion are predicted using artificial neural networks (ANN) trained with a database constructed from finite element method (FEM) simulations. Three different cases are optimized varying the carbon fibre layers orientations: $-45^{\circ} / 45^{\circ}, 0^{\circ} / 90^{\circ}$, and a case with the two-fibre layer orientations intercalated. Five geometric parameters and a number of carbon fibre layers are selected as design parameters. A sensitivity analysis is performed using the Garzon equation. The best balance between displacement and failure criterion was found with fibre layers oriented at $0^{\circ} / 90^{\circ}$. The optimal candidate with $-45^{\circ} / 45^{\circ}$ orientation presents higher displacement; however, the Tsai-Wu criterion was less than 0.5 and not suitable for RBP design. The case with intercalated fibres presented a minimal displacement being the stiffer RBP design. The damage concentrates mostly in the zone that contacts the ground. The sensitivity study found that the number of layers and width were the most important design parameters.

Keywords: optimization, crow search algorithm, artificial neural networks, running blade prosthetics, Tsai-Wu criterion, finite element method
Highlights
- $\quad$ An optimization methodology was proposed for the RBP design.
- A new technique for RBP manufacturing was evaluated.
- Carbon fibre layers with layers oriented $0^{\circ} / 90^{\circ}$ were the best option for the design of the RBP.
- $\quad$ The proposed methodologies reduce the manufacturing costs of the RBP.

\section{O INTRODUCTION}

A prosthesis is an artificial element integrated into the human body to replace an internal or external organ. The most common prostheses are those that replace upper and lower limbs. With the use of new materials and better manufacturing techniques, it has been possible to optimize prosthetic models to replace amputated arms and legs [1] to [3]. Regarding leg prostheses, the applications are diverse; everyday cosmetic prostheses have been created that allow an amputated person to lead a normal life, thereby improving their living conditions. Furthermore, advanced prostheses have been developed that have allowed an athlete with amputations in one or both lower limbs took take part in sports activities almost at the level of a non-amputee athlete. An example is the blade type prostheses used by the South African athlete Oscar Pistorius made with carbon fibre, a composite material with applications in the automotive and aerospace industry, which has properties similar to steel but with little weight, which makes it ideal for prosthetic applications. Manufacturers of these sports implement, for low and high impact activities, such as Ösur and Ottobock, offer various blade-type sports prostheses, but the high cost of these makes them inaccessible to the general public [4]

An alternative in the manufacture of sports prostheses, like running blade prosthetics (RBP), is the inclusion of additive manufacturing in the process of developing a human prosthesis, in particular, fused deposition modelling, which is one of the most widely used processes due to its simplicity and ease of operation. Therefore, the mechanical properties that composite materials offer can be combined with the advantages of fused deposition modelling, mainly in small-scale production, with the option of manufacturing highly personalized products and, at the same time, with shorter prototypes development times [5].

Türk et al. [6] evaluate the mechanical performance of lower limb prostheses manufactured combining additive manufacturing with carbon fibre-reinforced polymers. They found that by using this technique, the mechanical strength increases by around $40 \%$, and the weight decreases by $28 \%$.

Tavangarian et al. [7] studied the mechanical strength of the pylon section of a lower limb prosthetic 
manufactured with 3D printing using polylactic acid polymers. They found that this material meets the standards set by ISO 1038:2016 [8] regarding the structural requirements of lower-limb prostheses.

Ouarhim et al. [9] perform the characterization and numerical simulation of laminated glass fibrepolyester composites applied to running blade prosthetics. They used software to simulate the composite materials. Its results show that the number of layers impacts in bending and buckling. Its results show a good agreement between experimental and numerical.

A good balance between displacement and mechanical resistance guarantees the good performance of an RBP. During the change of length or displacement of an RBP, energy is stored and returned, increasing the forward propulsion [10]. However, increasing the displacement can lead to the mechanical failure of RBP, mainly in the sections with a curved shape.

To achieve an optimal design of an RBP, advanced tools as optimization algorithms are required. These types of algorithms are widely used to solve diverse problems in engineering fields [11] to [14]. A common characteristic of optimization algorithms is the large number of objective function evaluations required to find an optimal solution. When experimental or finite element method (FEM) calculations are employed to perform objective function evaluations, the computational cost increases, making the optimization process infeasible. In these cases, surrogated methods are an option to reduce the optimization time [15] to [17]. An example of an optimization strategy assisted by surrogated methods applied to lower limb prosthesis manufactured with composite materials can be found in [18], focusing on the optimization of the pylon tube made of isogrid structures. Due to optimization, they achieved a reduction of $13.3 \%$ and $70.59 \%$ in the mass and the Tsai-Wu index under compression effects.

Unlike previous optimization work, the present investigation is applied to a lower-limb sport prosthetics which can be used to reintegrate people with lower-limb amputations into sport activities. Also, optimization methodology employs artificial neural networks (ANN) with two intentions: to evaluate the objective function and to use the ANN coefficients in the calculation of relative importance of design parameters.

This work employs an optimization strategy to improve the design of an RBP. This strategy integrates crow search algorithm (CSA), and ANN and FEM calculations. The objective of the optimization was to find a design that has better resistance to failure without decreasing its capability to displace in order to maintain its functionality. The failure criterion used for determining the resistance of the RBP was the Tsai-Wu criterion. To reduce the computational cost, displacements and the Tsai-Wu failure criterion were calculated by means of ANN. The ANN was trained with a database constructed from FEM calculations of displacement and Tsai-Wu failure. The manufacturing process, modelled using commercial FEM software, is a lay-up process with carbon fibre layers stacked around acrylonitrile butadiene styrene (ABS) cores. Three carbon fibre orientations were tested to find the better design option: $-45^{\circ} / 45^{\circ}, 0^{\circ} / 90^{\circ}$, and a combined case with $-45^{\circ} / 45^{\circ}$ and $0^{\circ} / 90^{\circ}$ orientations. Using the ANN coefficients, a sensitivity analysis was performed to know the relative importance of the design parameters in each orientation.

\section{METHODS}

\subsection{RBP Design and Manufacturing}

An RBP is a hook-shaped artefact made primarily of composite materials from epoxy matrix carbon fibres. The manufacturing processes used for its manufacture are the lay-up process and the pre-impregnated fibre process. The first consists of applying or mixing epoxy resin with a brush or roller into the carbon fibre layers that are stacked until reaching the thickness based on the desired resistance. Another manufacturing process consists of the use of pre-impregnated carbon fibre layers that, after being stacked, must undergo a thermal treatment in an autoclave for the activation of the resin [19]. The RBPs have a spring-like mechanical behaviour; during the first gait cycle, the prosthesis is compressed by the effect of the dynamic load of the body storing energy. In the second stage of the gait cycle, that stored energy is released, generating a thrust forward, just as the heel joint of the human foot does. The maximum efficiency in this energy return of a high-performance RPB ranges between $63 \%$ and $95 \%$ of the accumulated energy [20].

\subsection{Optimization Methodology}

A bio-inspired method used in optimization is the CSA. This novel algorithm, developed by Askarzadeh [21], is based in the behaviour of crows, considered the most intelligent birds. A characteristic of the crow's behaviour is the way they store food in hideouts to prevent that other crows from stealing it; at the same time, they look for opportunities to steal 
the food of other members of the flock. Crows use the gained experience stealing food to deceive potential plunderers, employing diverse strategies [22]. Crows, always try to find optimal food places. This intelligent behaviour is replied in the CSA in which the optimal place is analogous to the global optima into the search space. In CSA, it is assumed that the crows live in flocks, can memorize the places where they hide their food, a crow follows another to try to steal its food and protect its hideouts from other crows via a probability reason [22].

In the CSA optimization process, during a iteration iter, a crow $i$, from a flock of size $N$, occupy a position defined by the vector $x^{i, i t}=\left[x_{1}^{i, i t}, x_{2}^{i, i t}, \ldots, x_{d}^{i, i t}\right]$, where $i=1,2, \ldots, N, \quad i t=1,2, \ldots, i t_{\max }, i t_{\max }$ is the maximum number of iterations, and $d$ is the dimension of the problem or the number of variables in the design. The position, $m^{i, i t}$, of the hideout it is stored in the crow memory and represents the best position at $i t$, the far. For the next iterations, the crow will try to find better positions.

Two situations may occur when, at iteration it, a crow $j$ visits the hideout where it stores its food and a crow $i$ is on the lookout attempting to steal it: (1) the crow $j$ does not realize that crow $i$ followed it and reveals its hideout to the thief crow; (2) the crow $j$ detects the presence of the crow $i$ and changes its trajectory to avoid revealing the location of its hideout. In the first situation, the new position of crow $i$ is defined by:

$$
x^{i, i t+1}=x^{i, i t}+r_{i} \times f l^{i, i t} \times\left(m^{i, i t}-x^{i, i t}\right),
$$

where $0 \leq r_{i} \leq 1$ is a random number with uniform distribution and $f l^{i, i t}$ is the flight length of crow $i$ at iteration it. If the second situation arises, the crow $j$ will change its trajectory through fake hideouts to deceive to crow $i$. Both situations are expressed as follows:

$$
x^{i, i t}=\left\{\begin{array}{cc}
x^{i, t}+r_{i} \times f l^{i, i t} \times\left(m^{j, i t}-x^{i, i t}\right) & r_{j} \geq A P^{j, i t} \\
\text { a random position } & \text { otherwise }
\end{array}\right.
$$

where $0 \leq r_{j} \leq 1$ is a random number with uniform distribution and $A P^{j, i t}$ is the awareness probability of the crow $j$ at iteration it. Intensification and diversification are two important characteristics of metaheuristic algorithms; these characteristics in CSA are provided by $f l$ and $A P$. Lower values of both parameters will guide the optimization to local search, increasing the intensification, whereas larger values expand the search randomly in the decision space but reduce the probability of found optimal solutions, resulting in increasing diversification.

In Fig. 1, the flowchart of the CSA is presented. In the first step, the objective function, the range of decision variables and constraints are defined, also, the CSA parameters, $N, i t_{\max }, d, f l$, and $A P$ are initialized. In Step 2 the positions of flock crows are defined randomly; because the crows do not have prior experience at Iteration 1, it is assumed that the initial position is the location of the hideout. In Step 3 , the algorithm evaluates the objective function. In the next step, all the crows of the flock update its position using the next procedure: a crow $i$ randomly selects a crow $j$ of the flock and tries to discover the position of the hideout, $m^{j}$. The position is updated using Eq. (2). In Step 5, if the new position of crow $i$ is feasible, then its position is updated; if not, crow $i$ will remain at the initial position. In the next step, the objective function is evaluated for the new crow flock positions. In Step 7, the memory of the crow flocks is updated. A comparison is made between the value of the objective function evaluated with both the new position $x^{i, i t+1}$ and the position $m^{i, i t}$. If the new position performs better. the memory it is updated; if not, the memory remains without changes. This is mathematically stated as follow:

$$
m^{i, i t+1}=\left\{\begin{array}{cc}
x^{i, i t+1} F_{o b j}\left(x^{i, i t+1}\right) \text { better than } F_{o b j}\left(m^{i, i t+1}\right) \\
m^{i, i t+1} \text { otherwise }
\end{array} .\right.
$$

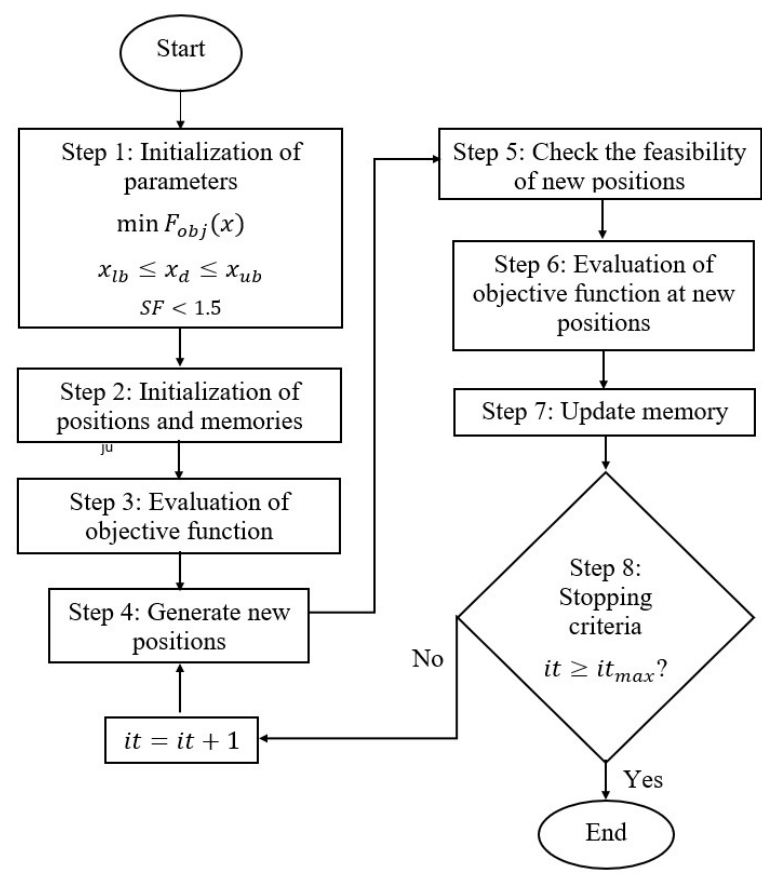

Fig. 1. CSA flowchart 


\subsection{Artificial Neural Network}

An ANN is a machine-learning technique used in the prediction of output variables as a function of input variables. A typical ANN consists of processing elements or neurons grouped in layers sequentially connected by synaptic weights. The most popular type of ANN is the multi-layer perceptron (MLP) with at least three neurons layers divided into input, hidden, and output layers. The number of neurons clustered at input and output layers is equal to the number of input and output variables, respectively, whereas, in the case of the hidden layer, the number of neurons is defined in terms of the predictive accuracy level of the ANN model [23] to [25]. The output of layers is computed by means of transfer functions whose argument is the summation of the multiplication of the corresponding weight by input signals plus a bias [26] a comprehensive review of the artificial neural network (ANN. The ANN prediction is computed by the next expression:

$$
A N N_{\text {output }}=T F^{O}\left[\sum_{j=1}^{n}\left(w_{j}^{2} T F_{j}^{H}\right)+b^{2}\right] .
$$

Here $A N N_{\text {output }}$ is the $\mathrm{ANN}$ prediction, $T F^{O}$ is the transfer function of the output layer, $w$ is the connection weight, $T F^{H}$ is the transfer function of the hidden layer, $b$ is the bias, $n$ is the number of neurons in the hidden layer, and the superscript 2 indicates the output layer.

The ANN is trained using a set of sample data. During the training, the ANN coefficients, $w$ and $b$, are proposed and the error between $A N N_{\text {output }}$ and real outputs, stored in a database, is calculated. This error is minimized by adjusting the coefficients by means of a training algorithm.

\subsection{Tsai-Wu Failure Theory}

The Tsai-Wu failure [27] theory is widely used in the prediction of failure in laminates. In the case of laminate analysis, the governing equation in matrix form is:

$$
\left\{\begin{array}{l}
N \\
M
\end{array}\right\}=\left[\begin{array}{ll}
A & B \\
B & D
\end{array}\right]\left\{\begin{array}{c}
\varepsilon^{0} \\
k
\end{array}\right\} .
$$

Here, $N$ and $M$ are the resultant force and moment respectively, $\varepsilon^{0}=\left[\begin{array}{c}\varepsilon_{x}^{0} \\ \varepsilon_{y}^{0} \\ \gamma_{x y}^{0}\end{array}\right]$ and $k=\left[\begin{array}{c}k_{x} \\ k_{y} \\ k_{x y}\end{array}\right]$ are the displacement in the midplane and curvature effects respectively, $A, B$ and $D$ are the extensional, coupling and bending matrices, which are calculated as follow:

$$
\begin{aligned}
& A=\sum_{k=1}^{n}\left(\overline{\mathbf{Q}_{\mathbf{k}}}\right)\left(t_{k}-t_{k-1}\right), \\
& B=\sum_{k=1}^{n}\left(\overline{\mathbf{Q}_{\mathbf{k}}}\right)\left(t_{k}^{2}-t_{k-1}^{2}\right), \\
& D=\sum_{k=1}^{n}\left(\overline{\mathbf{Q}_{\mathbf{k}}}\right)\left(t_{k}^{3}-t_{k-1}^{3}\right) .
\end{aligned}
$$

Here, $n$ is the total number of layers, $\overline{\mathbf{Q}}$ is the transformed stiffness matrix, and $t$ is the thickness of the composite layer. Using the stress-strain relationship for laminates, it is possible to calculate the state of stress of each composite layer, as is stated in Eq. (9):

$$
\left[\begin{array}{c}
\sigma_{x} \\
\sigma_{y} \\
\tau_{x y}
\end{array}\right]=\left[\begin{array}{lll}
\overline{\mathbf{Q}}_{11} & \overline{\mathbf{Q}}_{12} & \overline{\mathbf{Q}}_{16} \\
\overline{\mathbf{Q}}_{12} & \overline{\mathbf{Q}}_{22} & \overline{\mathbf{Q}}_{11} \\
\overline{\mathbf{Q}}_{16} & \overline{\mathbf{Q}}_{26} & \overline{\mathbf{Q}}_{66}
\end{array}\right]\left[\begin{array}{c}
\varepsilon_{x}^{0} \\
\varepsilon_{y}^{0} \\
\gamma_{x y}^{0}
\end{array}\right],
$$

where $\sigma_{x}$ and $\sigma_{y}$ are the normal stresses in $x$ and $y$ directions respectively, $\tau_{x y}$ is the shear stresses and $\gamma_{x y}^{0}$ is the shear strain. The calculation of the stress state allows using the Tsai-Wu failure criterion:

$$
H_{1} \sigma_{1}+H_{2} \sigma_{2}+H_{6} \tau_{12}+H_{11} \sigma_{1}^{2} H_{22} \sigma_{2}^{2}+H_{66} \tau_{12}^{2}<1 \text {. }
$$

The Tsai-Wu criterion states that the failure occurs when this condition is not satisfied. In the FEM software used, this condition is associated with the $S F$ in composite materials so that in FEM simulations, if $S F<1$ the failure is expected.

\section{PROBLEM FORMULATION}

This work focuses on obtaining the optimal design variables that allow the maximum displacements of an RBP constrained by the safety factor $(S F)$ based on the Tsai-Wu failure theory. The process used in the manufacturing of the RBP is the lay-up process, in which layers of $3 \mathrm{k}$ woven carbon fibre and epoxy resin are used. The fibre layers are placed in $-45^{\circ} / 45^{\circ}$ and $0^{\circ} / 90^{\circ}$ orientations.

The strategy employed consists of obtaining the design parameters through an optimization algorithm that evaluates the objective function that defines the optimization problem; nevertheless, during the optimization, it is not feasible to evaluate it by means of numerical methods due to excessive consumption of time and computational resources. To improve the optimization process, a mathematical model is 
constructed using a surrogated method. This model will predict both the displacement and SF, which are required in objective function evaluations. To obtain this model, it is necessary to construct a database that contains a set of combinations of design parameters with their respective responses, in this case, displacement and $S F$. The responses to be obtained from FEM calculations due to the number of required simulations is significantly lower than in the optimization process. Employing this strategy led to a reduction of the required time to design an RBP. For this research, the chosen optimization method was the CSA, whereas ANN was used as surrogated method.

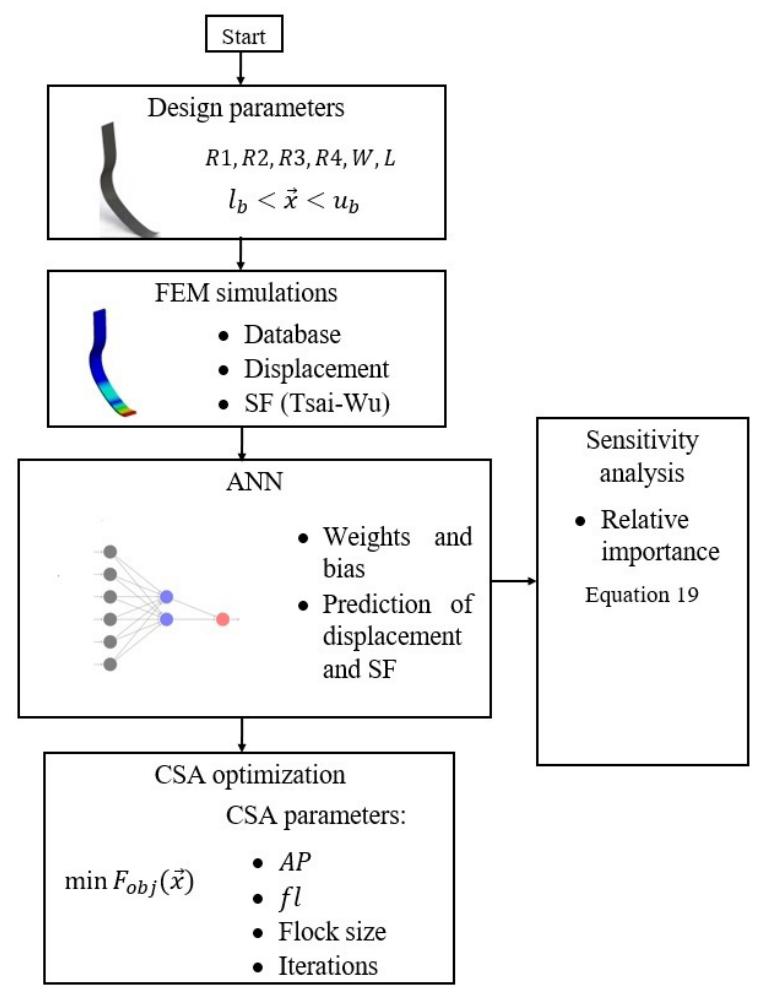

Fig. 2. Optimization procedure

The sequence of activities to optimize the RBP is described in Fig. 2. First are defined the design variables with its bounds and the prothesis load conditions. Afterward, the FEM calculations are performed to generate the database whose combinations are based on a design of experiments in order to promote the learning of the ANN. In the next step, the database was used to train and test the ANN models that will predict the prosthesis displacements and the $S F$, respectively. In this step, the ANN coefficients, weights, and biases are obtained. Before the optimization, the relative importance of each of the design variables in the variables predicted is calculated by means of a sensitivity analysis. Finally, in the optimization process, the CSA parameters are defined, and the objective function is evaluated, obtained as a result the optimization of the RBP. This process is performed three times for each of the fibre layer orientations. A detailed description of this strategy is presented in the next sections.

\subsection{CSA Parameters}

Three optimizations were performed for different layer orientations: a first case, $\mathrm{C} 1$, with layers of carbon fibre with orientation $-45^{\circ} / 45^{\circ}$, a case $\mathrm{C} 2$ with layers of carbon fibre with orientation $0^{\circ} / 90^{\circ}$ and a third case, $\mathrm{C} 3$, with a combination of carbon fibre layers oriented $-45^{\circ} / 45^{\circ}$ and $0 / 90^{\circ}$. The objective function that defines the problem is expressed by:

$$
\min F_{o b j}=15-d_{A N N},
$$

where $F_{o b j}$ is the objective function, $d_{A N N}$ is the displacement calculated by means of ANN and 15 is a maximum displacement, expressed in $\mathrm{mm}$, expected during the optimization. This value was set based on the results obtained from the central composite design of experiments. The objective function must be minimized because it is expected that as the obtained displacement increases during CSA iterations, the value of the function approaches zero. The objective function is subjected to:

$$
\begin{gathered}
1.2-S F_{A N N} \leq 0, \\
x_{i}^{l b} \leq x_{i} \leq x_{i}^{u b},
\end{gathered}
$$

here $S F_{A N N}$ is the safety factor predicted using ANN, $x_{i}$ is the $i^{\text {th }}$ design variable, and the superscripts $l b$ and $u b$ are the lower and upper bound, respectively.

The same optimization parameters were used for the three optimization cases. Each optimization was carried on during 1000 iterations; the flock size was 50 crows, $f l$ was 2 , and $A P$ was 0.1 .

\subsection{Parameterization}

In Fig. 3, the geometric segments of the RBP profile are illustrated. For the optimization, the dimensions of two of them stay constant. These two segments belong to the joint with the human body (top of the RBP), with a length of $97.12 \mathrm{~mm}$, and the contact area with the ground (bottom of the RBP), with an arc length of $10 \mathrm{~mm}$. The remaining four segments, associated with the letter R, are defined geometrically by radii whose dimensions were selected as design parameters for the optimization. To guarantee a smooth transition, the 
points of segments $R 1, R 2, R 3$ and $R 4$ are tangential to each other. The last geometric parameter corresponds to the width, $W$, of the RBP. An additional parameter is the number of layers of carbon fibre that cover the ABS core. This parameter must be an even number to maintain the same number of layers on both the front and the back of the RBP. The bounds of the design parameters were the same for the three optimization cases and are listed in Table 1. The geometry used was a surface modelled in CAD software, based on the dimensions of a commercial prosthesis [28]. The geometry profile was obtained from the centreline of the original one, as it was required to generate the surface necessary for the simulation of a FEM module for composite materials.

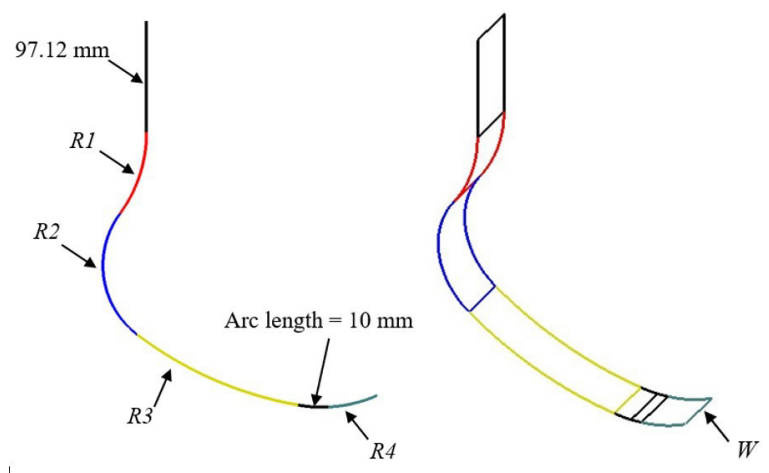

Fig. 3. Geometrical parameters of the RBP

Table 1. Design parameter bounds

\begin{tabular}{lcc}
\hline Design parameter & Lower bound $(l b)$ & Upper bound $(u b)$ \\
\hline$R 1[\mathrm{~mm}]$ & 106.2 & 129.8 \\
\hline$R 2[\mathrm{~mm}]$ & 74.7 & 91.3 \\
\hline$R 3[\mathrm{~mm}]$ & 283.5 & 343.5 \\
\hline$R 4[\mathrm{~mm}]$ & 110.7 & 135.3 \\
\hline$W[\mathrm{~mm}]$ & 40.5 & 49.5 \\
\hline No. of layers [-] & 18 & 26 \\
\hline
\end{tabular}

\subsection{Generation of Database and FEM Simulations}

For each optimization case, a database based on a central composite design of experiments was generated to be used in the ANN predictions. The size of each database was $6 \times 125$, where 6 is the number of input variables or design variables and 125 is the number of design combinations in the database. The dimensions of each combination varied according to the design of experiments, except for those remining constant, as was referred in Fig. 3. For each combination, the displacement and the SF using static linear FEM calculations were calculated. To characterize the materials in the simulations, a special module for composite materials was used. Here, were set the inputs shown in Table 2; the number of layers, in even number, to maintain the symmetry of the prosthesis, the materials that make up the ABS composite material, fibre, core, and combinations of fibre orientations. Properties of carbon fibre and ABS are presented in Tables 3 and 4, respectively.

Table 2. Input parameters for composite materials

\begin{tabular}{lc}
\hline Fabrics & ABS, Core \\
\hline Number of layers & $18,20,22,24,26$ \\
\hline Sub laminates & $-45^{\circ} / 45^{\circ}, 0^{\circ} / 90^{\circ}$ \\
\hline
\end{tabular}

Table 3. Properties of $3 k$ woven carbon fibres

\begin{tabular}{lcc}
\hline Properties & Unit & Value \\
\hline$E_{x}$ & {$[\mathrm{MPa}]$} & 59160 \\
\hline$E_{y}$ & {$[\mathrm{MPa}]$} & 59160 \\
\hline$E_{z}$ & {$[\mathrm{MPa}]$} & 7500 \\
\hline$v_{x y}$ & {$[-]$} & 0.04 \\
\hline$v_{y z}$ & {$[-]$} & 0.3 \\
\hline$v_{x z}$ & {$[-]$} & 0.3 \\
\hline$\rho$ & {$\left[\mathrm{kg} / \mathrm{m}^{3}\right]$} & 1451 \\
\hline
\end{tabular}

Table 4. Properties of ABS

\begin{tabular}{lcc}
\hline Property & Unit & Value \\
\hline$E$ & {$[\mathrm{MPa}]$} & 2588 \\
\hline$v$ & {$[-]$} & 0.36 \\
\hline$\rho$ & {$\left[\mathrm{kg} / \mathrm{m}^{3}\right]$} & 1040 \\
\hline
\end{tabular}

This configuration was exported to a static structural analysis, where each layer was discretized using shell elements, which have 4 nodes per element and 6 degrees of freedom per node. The finite elements have a size of $2 \mathrm{~mm}$ resulting in a mesh size of 6400 elements per layer (Fig. 4a). The number of elements was selected based on the comparison between FEM and experimental measurements.

The load was applied in the top segment of the RBP, whereas the displacement was constrained in all directions in the segment of contact with the ground (Fig. 4b). The magnitude of the load was set considering a human weight of $77.6 \mathrm{~kg}$, which results in a static load of $761.25 \mathrm{~N}$. For dynamic conditions, the load was increased 2.7 times, which corresponds to a maximum load peak during running [29]. Then, the applied load was $2055 \mathrm{~N}$. 

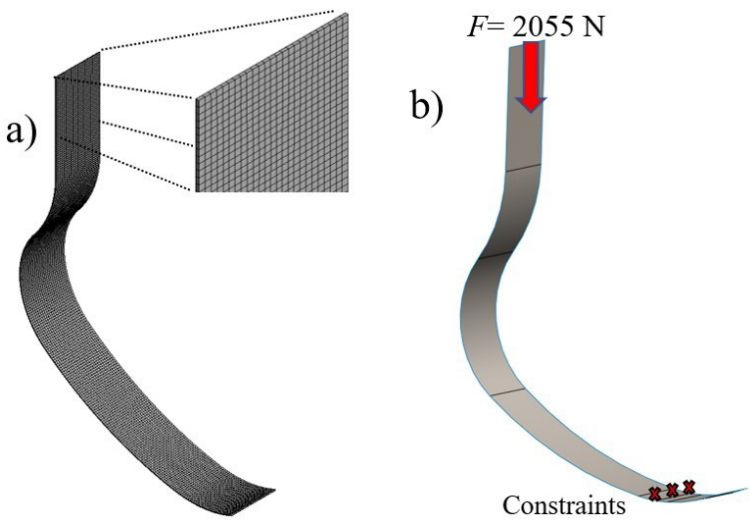

Fig. 4. a) Discretized model, and b) force and constraints

From the FEM simulations, the displacement of RBP and the SF based on Tsai-Wu failure theory were obtained.

\subsection{ANN Modelling}

To reduce the computational time during the design optimization, a total of six ANN models were trained to predict the displacement and SF factor for each of the optimization cases. The ANN was trained using the Levenberg-Marquardt algorithm [30] and [31] with a learning rate of $1 \times 10^{-4}$. The transfer function for the hidden layer, $T F^{H}$, was the hyperbolic transfer function Eq. (14), whose argument is defined by Eq. (15), whereas output layer $T F^{O}$ was linear [32] and [33].

$$
\begin{gathered}
T F_{j}^{H}=\frac{2}{1+e^{-2 x_{j}}}-1, \\
x_{j}=\sum_{i=1}^{m}\left(w_{i}^{1} i n_{i}\right)+b_{n}^{1},
\end{gathered}
$$

Here $x_{j}$ is the argument for the transfer function of the $j^{\text {th }}$ hidden neuron, $m$ is the number of neurons in the input layer or number of inputs and the superscript 1 indicates the hidden layer.

The architecture of the net consists of an input layer with six neurons and one neuron at the output layer. In the hidden layer, all the nets that were used to predict the displacement and Tsai-Wu criterion in the optimization cases contain two neurons.

Eighty per cent of data samples were used for training, and the rest for testing. Input data were normalized between 0.1 and 0.9 by means of [34]:

$$
i n_{i}=0.8\left(\frac{I n_{i}-I n_{\text {low }}}{I n_{\text {upp }}-I n_{\text {low }}}\right)+0.1 \text {, }
$$

where $i n_{i}$ is the normalized input, $I n_{i}$ is the unnormalized input, $I n_{\text {low }}$ is the input lower bound and $I n_{u p p}$ is the input upper bound.

During training and testing, the ANN was evaluated using the mean squared error, and the correlation coefficient defined by Eqs. (17) and (18), respectively:

$$
\begin{gathered}
R M S E=\frac{\sum_{t=1}^{T}\left(y_{A N N}-y_{F E M}\right)^{2}}{T}, \\
R^{2}=1-\frac{\sum_{t=1}^{T}\left(y_{A N N}-y_{F E M}\right)^{2}}{\sum_{t=1}^{T}\left(y_{F E M}-y_{\text {vve }}\right)},
\end{gathered}
$$

where $y_{A N N}$ is the output predicted by ANN, $y_{F E M}$ is the output predicted by FEM, $y_{\text {ave }}$ is the average of actual values and $T$ is the number of samples.

\subsection{Sensitivity Analysis}

A sensitivity analysis enables determining the relative importance of each of the design variables in the displacement and in the Tsai-Wu criterion. For this purpose, the equation proposed by Garson [35] was used. Eq. (19) is suitable due its use of the ANN synaptic weights to calculate the percentage of importance of each variable. In addition to relative importance, the obtained results of the sensitivity analysis can provide information about of the relation between the design variables.

$$
I_{m}=\frac{\sum_{j=1}^{n}\left(\frac{\left|w_{m n}^{1}\right|}{\sum_{i=1}^{m}\left|w_{i n}^{1}\right|} \times w_{j m}^{2}\right)}{\sum_{i=1}^{m}\left(\sum_{j=1}^{n} \frac{\left|w_{i n}^{1}\right|}{\sum_{i=1}^{m}\left|w_{i n}^{1}\right|} \times w_{j m}^{2}\right)},
$$

\section{RESULTS}

\subsection{Numerical Validation}

A comparison was made between FEM simulations and experimental test results reported by RoselSolis et al. [36]. The specimen was manufactured by the lay-up process with carbon fibres with $0^{\circ} / 90^{\circ}$ orientation. The machine was a Shimadzu AG-IC stress floor machine with a load cell of $100 \mathrm{kN}$. The material conditions were the same as described above, whereas the force and support conditions were set as in the experiment. The comparison is shown in Fig. 
5. The maximum difference between experimental and numerical displacements was around $8 \%$ caused by a force near $600 \mathrm{~N}$. From there, the difference decreases considerably. Based on these results, it can be stated that the proposed methodology to calculate displacements and SF is highly suitable for this purpose.

\subsection{ANN Results}

Six models were trained to vary the number of hidden neurons to obtain the best ANN performance. The architecture obtained was 6:2:1 for all ANN models. This means that two neurons are in the hidden layer. The comparison of FEM and ANN results for displacement and $S F$ are presented in Fig. 6. A good approach is observed in the prediction of both variables. The values of $R^{2}$ were $>0.99$ for all models, whereas the values obtained of $M S E$ were $<1.3 \times 10^{-2}$.

a)

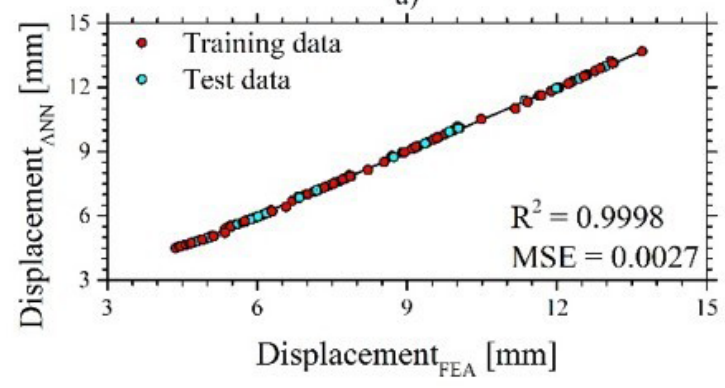

c)

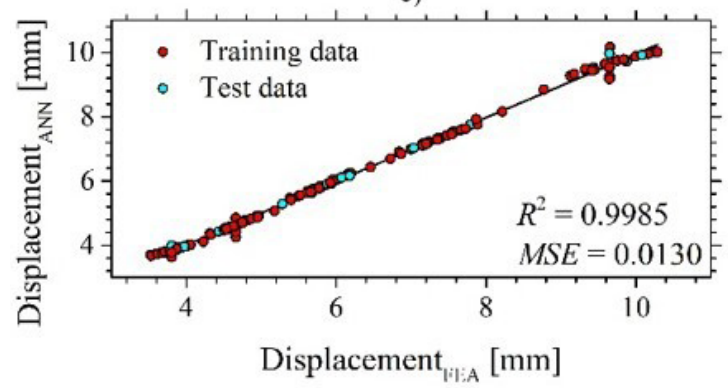

e)

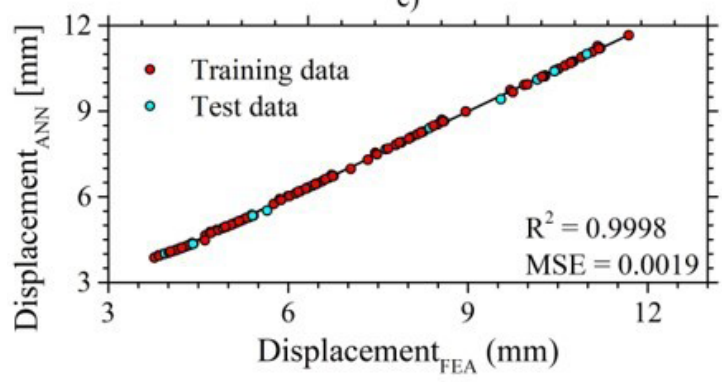

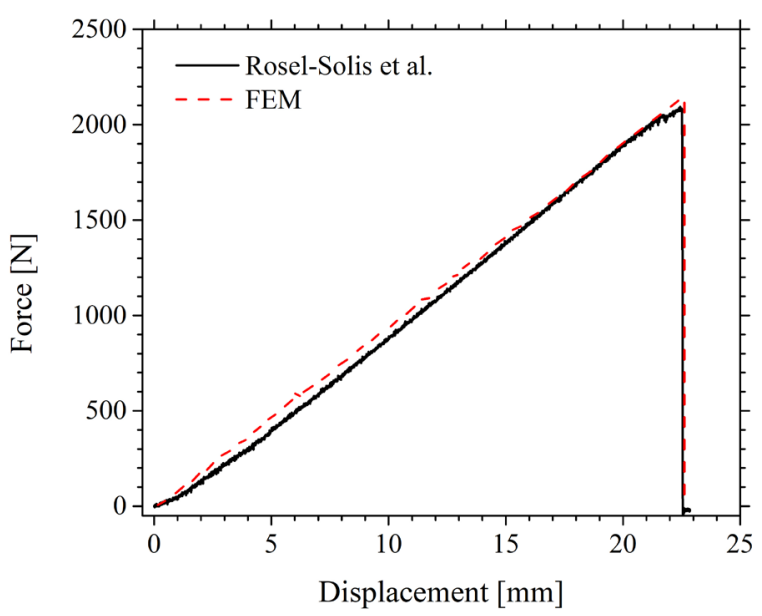

Fig. 5. Comparison between experimental and numerical results

Five new FEM simulations, with different design parameters than the stored in database, were performed

b)

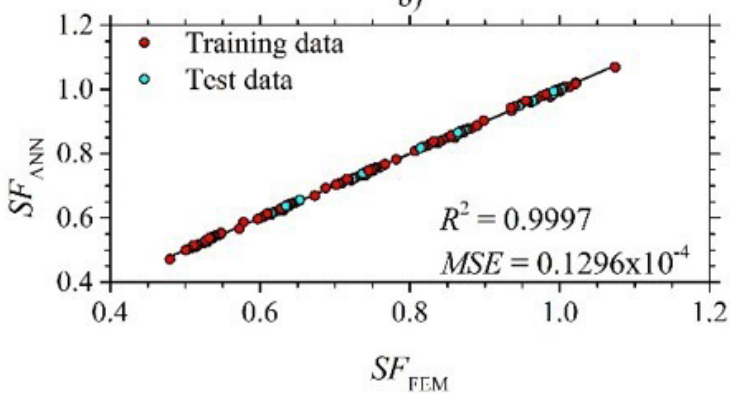

d)

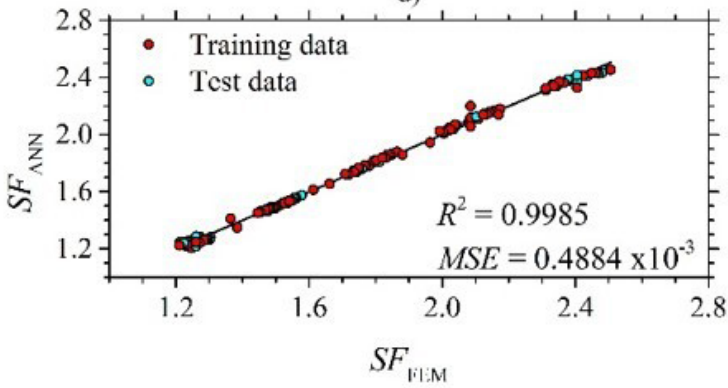

f)

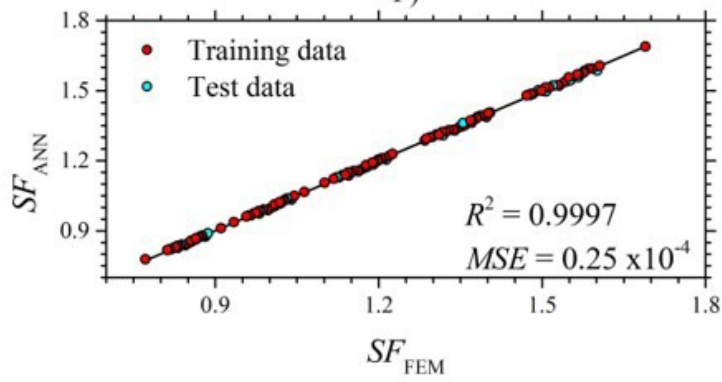

Fig. 6. Comparison of ANN and FEM predictions; a) C1 displacement, b) C1 SF, c) C2 displacement, d) C2 SF, e) C3 displacement, f) C3 SF 
to test each ANN models. The results are presented in Fig. 7. The prediction of displacement and SF in $\mathrm{C} 1$ had an average error of $5 \%$ and $2 \%$, respectively. For $\mathrm{C} 2$, the average error for displacement was 3.5 and for SF was $3.2 \%$, whereas in $\mathrm{C} 3$ the ANN model predicted the displacement and SF with an average error of $4.1 \%$ and $4.2 \%$, respectively. These findings guarantee the capability of ANN accurate predictions with the proposed architecture.

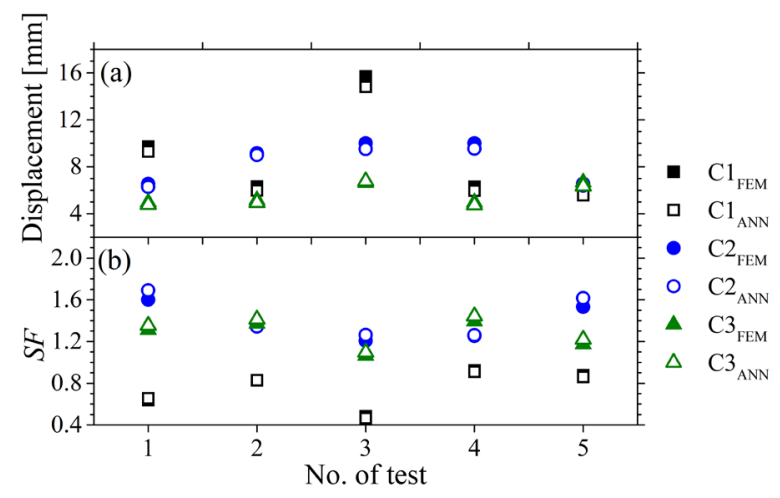

Fig. 7. Testing of ANN models

\subsection{Optimization Results}

The optimization convergence for three cases is presented in Fig. 8. Around the first 300 iterations, the CSA has identified the region of the search space where the optimal solutions are located. Minimal changes are observed in the objective function value from the subsequent iterations.

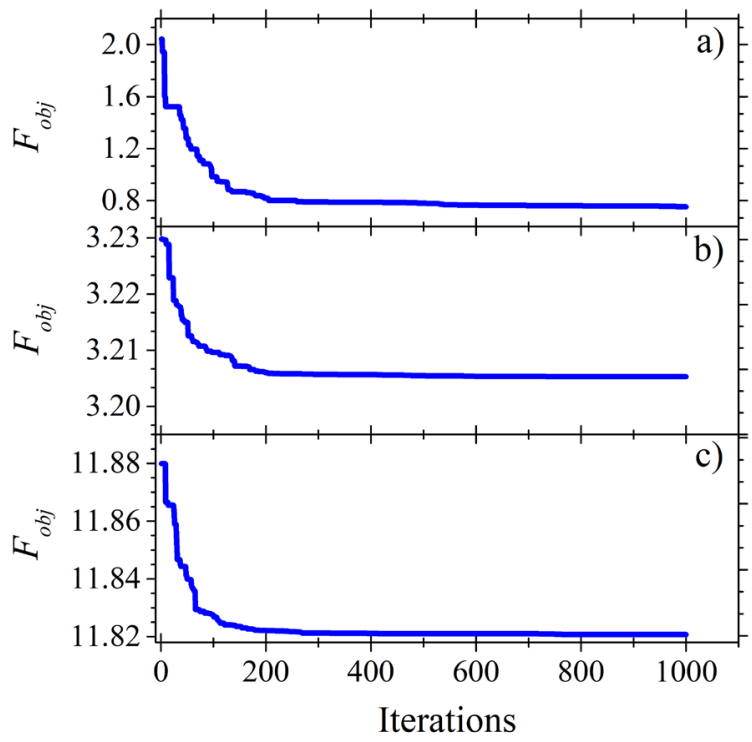

Fig. 8. CSA optimization convergence for: a) $\mathrm{C} 1$, b) $\mathrm{C} 2$, and c) $\mathrm{C} 3$
The optimized results of displacement and SF are presented in Table 5. In $\mathrm{C} 1$, with fibres oriented $-45^{\circ} / 45^{\circ}$, no solutions that meet the requirements of an $S F>1.2$ were found; however, the displacement was higher than in the rest of the cases. This result shows that the fibre orientation is not suitable to resist the working conditions of the RBP. The C3 presents the higher $S F$, but at the same time, the displacement was shorter with only $5 \mathrm{~mm}$. The best balance between displacement and $S F$ was found in C2. In this case, the displacement was 9.19, and the SF was 1.34. Carbon fibres with $0^{\circ} / 90^{\circ}$ orientation results in the best option for the design of RBP. The addition of layers with $0^{\circ} / 90^{\circ}$ in a combined case, $\mathrm{C} 3$, reduces the capability of displacement of only layers oriented $-45^{\circ} / 45$. The fibres oriented at $-45^{\circ} / 45^{\circ}$ have a more elastic behaviour because they are not perpendicular to the direction of the applied load; therefore, there is energy dissipation.

Table 5. Displacement and SF obtained from optimization

\begin{tabular}{lcl}
\hline & Displacement $[\mathrm{mm}]$ & $S F$ \\
\hline $\mathrm{C} 1$ & 16.24 & 0.43 \\
\hline $\mathrm{C} 2$ & 9.19 & 1.34 \\
\hline $\mathrm{C} 3$ & 5 & 1.45 \\
\hline
\end{tabular}

In Fig. 9, the displacement and SF obtained from the optimization were compared with those obtained in the database. The search space is reduced to the zone where one of the two requirements, either displacement or $S F$, stands a greater chance of improvement. In $\mathrm{C} 1$, the increase of displacement is remarkable due to the low capability of RBP design to increase its strength to the applied load. C2 presents the same trend to improve its displacement; however, its $S F$ is over the defined limit, which favours the RBP design. In the case of $\mathrm{C} 3$, the trend is reversed and now the optimization leads toward and improvement of the $S F$, decreasing considerably its displacement. These results confirm $\mathrm{C} 2$ as the best option for the design of the RBP.

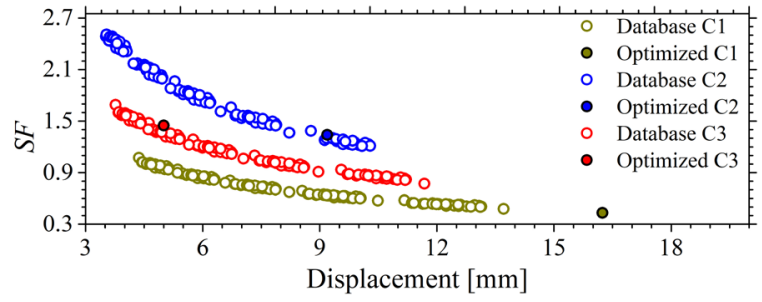

Fig. 9. Comparison between database and optimized cases; $\mathrm{C} 1, \mathrm{C} 2$, and $\mathrm{C} 3$ 
The design variables for each case are presented in Table 6. It is observed that R2 presents the same value for the three cases and corresponds to its lower bound. It should be noted that $W$ in $\mathrm{C} 1$ is on the low bound, and this had a negative impact in its resistance. Cases with a short number of layers were those who presented higher displacements, whereas in $\mathrm{C} 3$ with 24 layers, the displacement was reduced. Then, it is observed that a lower number of layers helps to decrease the stiffness. The R2 segment, being in the middle part of the RBP in relation to its height and a greater horizontal distance from the direction of application of the load, is subjected to a greater bending moment than the rest of the segments of the RBP. For this reason, the smaller the dimension of $\mathrm{R} 2$, the better the behaviour of the prosthesis. The values of segments R1, R3, and R4 vary depending on maintaining an optimal horizontal extension between $\mathrm{R} 2$ and R4 to minimize the effect of total flexion of the RBP. Also, the fact that manufacturing costs are reduced with fewer layers must be considered.

Table 6. Optimized design parameters

\begin{tabular}{lccc}
\hline Design parameter & C1 & C2 & C3 \\
\hline$R 1[\mathrm{~mm}]$ & 106.207 & 129.7 & 108.85 \\
\hline$R 2[\mathrm{~mm}]$ & 74.7 & 74.7 & 74.7 \\
\hline$R 3[\mathrm{~mm}]$ & 346.49 & 305.61 & 333.68 \\
\hline$R 4[\mathrm{~mm}]$ & 133.75 & 129.14 & 110.7 \\
\hline$W[\mathrm{~mm}]$ & 40.5 & 49.48 & 49.5 \\
\hline No. of layers & 18 & 18 & 24 \\
\hline
\end{tabular}

A comparison of the RBP profiles is presented in Fig. 10. At the segment R1, the profile of both $\mathrm{C} 1$ and $\mathrm{C} 3$ is similar and changes from R2, whereas the C2 profile presents lower outward curvature. All the profiles match in the junction of the end of R3, and the start of the ground contact area due to the length of this area is constant. Beyond the contact area, the profiles separate again, and the $\mathrm{C} 3$ curves inward to RBP more than $\mathrm{C} 2$ and $\mathrm{C} 3$.

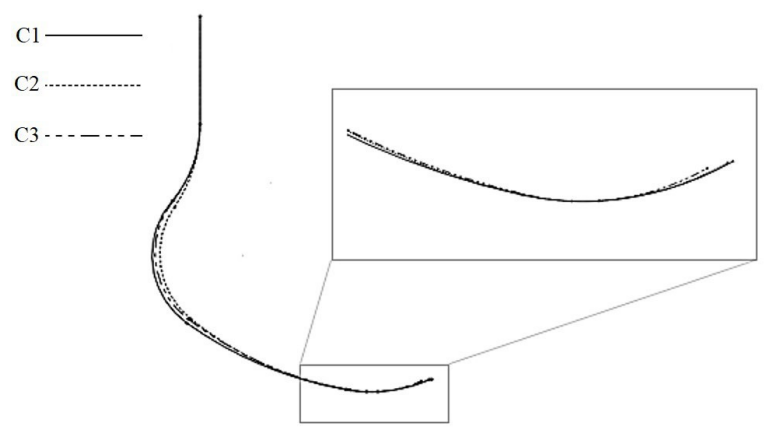

Fig. 10. Comparison of optimized RBP profiles
The displacement contours of the optimized RBP are presented in Fig. 11. The RBP displacement is higher at the top and diminishes until the area of contact with the ground. Beyond this point until the bottom tip, the displacements are negligible. The difference of displacements between top and point of contact of the RBP optimized models indicates that the stiffness of $\mathrm{C} 1$ design is lower than $\mathrm{C} 2$ and $\mathrm{C} 3$, whereas C3 presents higher stiffness. Higher displacement is a characteristic that favours the return of energy that the RBP must offer when operating. The $\mathrm{C} 1$ design presents the highest displacement value due to the behaviour of the fibres oriented at $-45^{\circ} / 45^{\circ}$ that tend to dissipate energy. This level of displacement would be desirable if it is complemented by an acceptable SF. The C2 design with fibre orientations at $0^{\circ} / 90^{\circ}$ offers a higher level of stiffness, which produces a lower displacement value than the $\mathrm{C} 1$ design; this is because part of the fibres of this configuration oppose resistance in a direction parallel to the applied force. The combination of $-45^{\circ} / 45^{\circ}$ and $0^{\circ} / 90^{\circ}$ orientations further increases stiffness, affecting displacement, obtaining the lowest displacement values of the three designs.

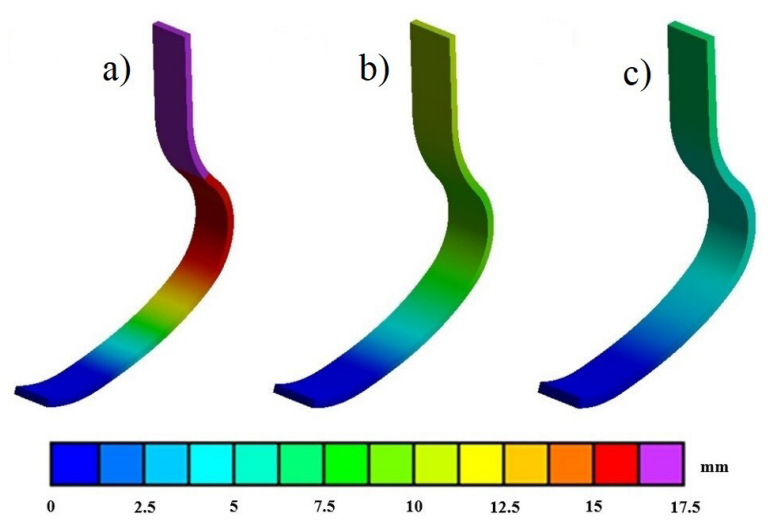

Fig. 11. Displacement contours of optimized cases;

a) $\mathrm{C} 1$, b) $\mathrm{C} 2$, and c) $\mathrm{C} 3$

Fig. 12 shows the zones where the SF occurs in the RBP optimized designs and its magnitudes. The critical zone is the contact area in which the $\mathrm{SF}$ is lower. This occurs due to the fact that in this segment the displacements are restricted, and reaction forces increases. Zones with high curvature also present lower SF, which can be attributed to a stress concentration in these locations. In $\mathrm{C} 1$, due to the orientation of layers, low SF magnitude covers a large section of the RBP. Areas below the minimum $(S F<1)$ appear in the $\mathrm{C} 1$ design; therefore, under the analysis conditions, it is not satisfactory since 
the RBP fails. The fibre orientation $-45^{\circ} / 45^{\circ}$ favours the flexural capacity of the RBP but acts negatively on its resistance. Designs C2 and C3 obtained satisfactory SF values, with $\mathrm{C} 3$ obtaining the best. However, considering the displacement results, the $\mathrm{C} 2$ design is the one that offers a better balance between displacement and $S F$, in addition to requiring a lower number of layers than $\mathrm{C} 3$. The combination of fibre orientation in the $\mathrm{C} 3$ design increased its stiffness and decreased its flexibility, obtaining a higher $S F$ but reducing displacement.

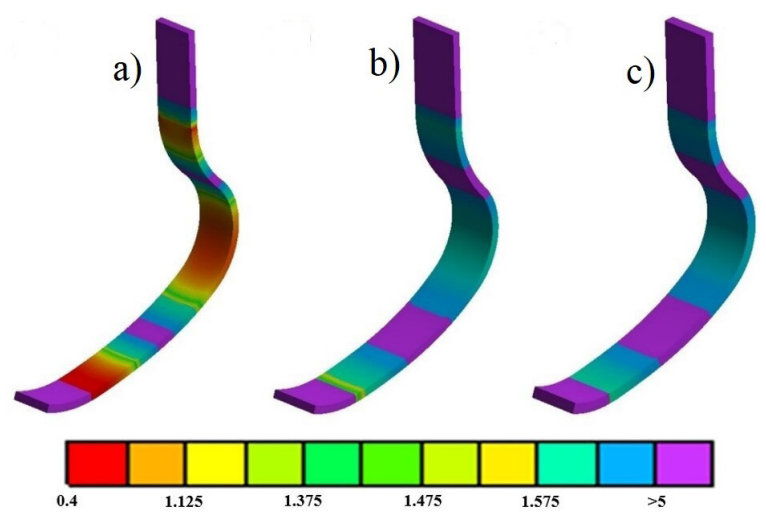

Fig. 12. SF contours of optimized cases; a) $\mathrm{C} 1$, b) $\mathrm{C} 2$, and c) $\mathrm{C} 3$

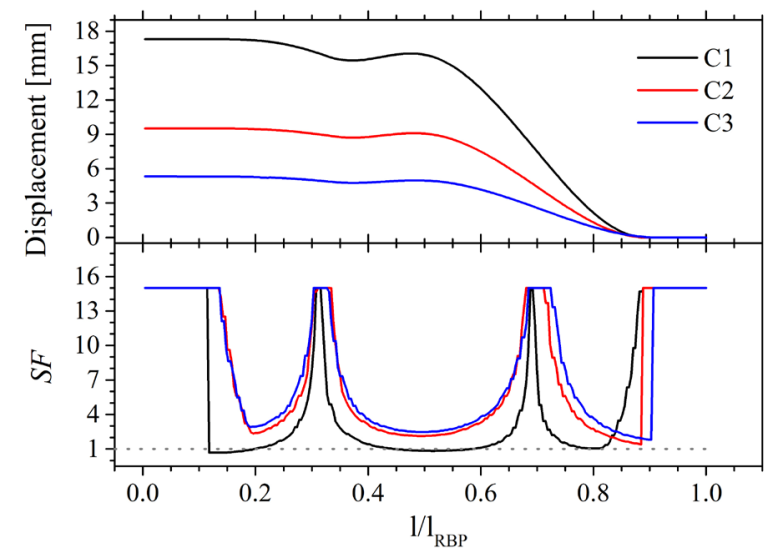

Fig. 13. Displacement and SF along RBP

Displacement and $S F$ were obtained along a path at the centre of the RBP in Fig. 13. The length of the RBP was normalized from top to bottom. The maximum displacement is observed at the top of the RBP and remains constant. Near the middle displacement diminishes and drops to zero at the point of contact. Due to the amplitude of displacements, large forward propulsion will be expected in $\mathrm{C} 1$ but, due to fluctuations observed, the return of energy will be smoother in $\mathrm{C} 2$ and $\mathrm{C} 3$. In the case of $S F$, three critical zones were identified. In the design, $\mathrm{C} 1$ failure occurs in almost $25 \%$ of the RBP length whereas in $\mathrm{C} 2$ and $\mathrm{C} 3$ the percentage of length with lower $S F$ values is smaller. Another difference was the region where minimum $S F$ appears in $\mathrm{C} 1$ is at $10 \%$ away from the RBP top; cases $\mathrm{C} 2$ and $\mathrm{C} 3$, this region was near the point of contact.

\subsection{Sensitivity Analysis Results}

The results of the sensitivity analysis are presented in Fig. 14. The displacement is mostly influenced by the number of layers in all cases and to a lesser extent by $\mathrm{R} 1$ for the cases $\mathrm{C} 1$ and $\mathrm{C} 3$, whereas in the $\mathrm{C} 2$ case, the lowest percentage of relative importance was the R4. In the $S F$, the number of layers is the most important variable for $\mathrm{C} 1$ and $\mathrm{C} 3$; however, in $\mathrm{C} 2$, it is the width of the prosthesis. These differences can be attributed to the orientation of the fibres. The fibres at $-45^{\circ} / 45^{\circ}$ make the RBP more flexible but decreases its resistance; therefore, to improve this negative condition, the carbon fibre layers must be increased. In the case of the $0^{\circ} / 90^{\circ}$ fibre orientation design, by increasing the width of the RBP, the number of fibres acting to support the applied load is increased, which improves their resistance and displacement.
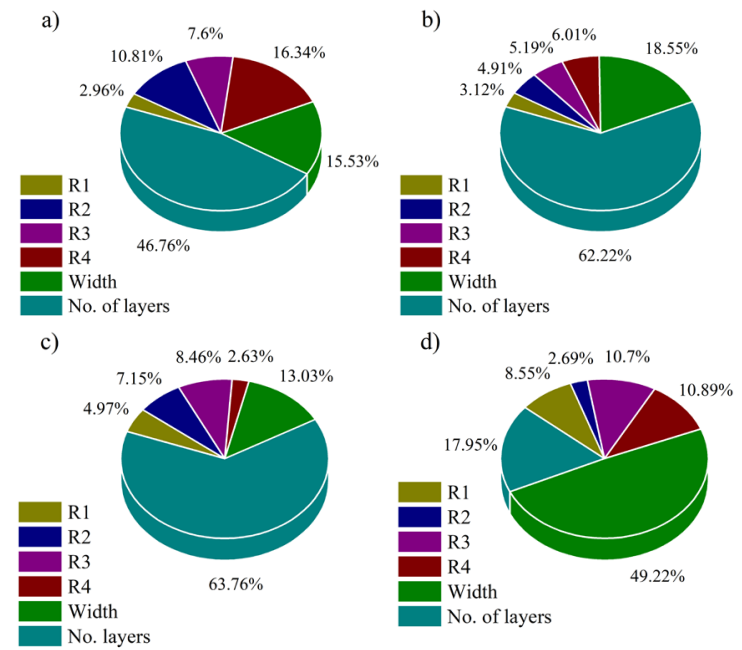

e)
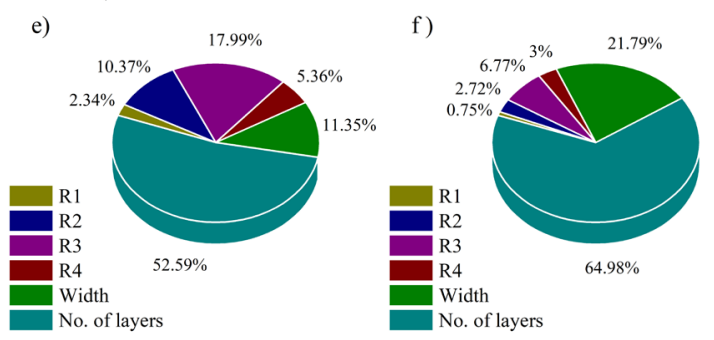

Fig. 14. Relative importance of the design parameters; a) C1 displacement, b) C1 SF, c) C2 displacement, d) C2 SF, e) C3 displacement, f) C3 SF 


\section{CONCLUSIONS}

An optimization procedure that includes CSA and ANN was employed to optimize the design of an RBP with the objective of increasing its displacement to prevent failure through the Tsai-Wu failure criterion. The RBP was made of carbon fibre layers stacked around a core made of ABS. Three carbon fibre layer orientations were tested. For the design of the RBP, the results show that the layers oriented at $0^{\circ} / 90^{\circ}$ represent the best trade-off between displacement and Tsai-Wu criterion with a reduced number of layers, whereas $-45^{\circ} / 45^{\circ}$ are not suitable due to imminent failure. The case with combined orientations presented minimal displacement, which would increase its stiffness. The damage was concentrated in the point of contact with the ground and, due to the selection of adequate design parameters, was minimized in the zones of the RBP with a curved shape. The two most important design parameters were the number of layers and the width of the RBP. The developed optimization procedure that combines CSA and ANN reveals a fast technique to improve the design of RBP; in addition, it is feasible to use the proposed manufacture technique for future orthopaedic personalized applications.

\section{REFERENCES}

[1] De Luigi, A.J., Cooper, A. (2018). Adaptive sports technology and biomechanics: Prosthetics. PM\&R, vol. 6, no. 8, p. S40-S57, D0l:10.1016/j.pmrj.2014.06.011.

[2] Monette, D., Dumond, P., Chikhaoui, I., Nichols P., Lemaire, E.D. (2020) Preliminary material evaluation of flax fibers for prosthetic socket fabrication. ASME Journal of Biomechanical Engineering, vol. 143, no. 2, p. 021006 , DOI:10.1115/1.4048079.

[3] Morais, D., Ferreira, Gomes, G., Silveira, M.E., Ancelotti A.C. (2019). Design optimization and development of tubular isogrid composites tubes for lower limb prosthesis. Applied Composite Materials, vol. 26, p. 273-297, D0l:10.1007/ s10443-018-9692-2.

[4] Marcellini, A., Ferez, S., Issanchou, D., De Léséleuc, E., Mc Namee, M. (2012). Challenging human and sporting boundaries: The case of Oscar Pistorius. Performance Enhancement \& Health, vol. 1, no. 1. p. 3-9, D0l:10.1016/j. peh.2011.11.002.

[5] Jin, Y., He, Y., Shih, A. (2016). Process planning for the fuse deposition modeling of ankle-foot-othoses. Procedia CIRP, vol. 42, p. 760-765, Dol:10.1016/j.procir.2016.02.315.

[6] Türk, D.-A., Einarsson, H., Lecomte, C., Meboldt, M. (2018) Design and manufacturing of high-performance prostheses with additive manufacturing and fiber-reinforced polymers. Production Engineering, vol. 12, p. 203-213, D0l:10.1007/ s11740-018-0799-y.
[7] Tavangarian, F., Proano, C., Zolko, C. (2019). Performance of low-cost 3D printed pylon in lower limb prosthetic device. TMS 2019 148th Annual Meeting \& Exhibition Supplemental Proceedings, The Minerals, Metals \& Materials Series, p. 1207-1215, D0I:10.1007/978-3-030-05861-6_115.

[8] ISO 10328:2016. Prosthetics - Structural Testing of LowerLimb Prostheses - Requirements and Test Methods. International standard organization, Geneva

[9] Ouarhim, W., Ait-Dahi, M., Bensalah, M.-Q., El Achaby, M., Rodrigue, D., Bouhfid, R., Qaiss, A. (2020). Characterization and numerical simulation of laminated glass fiber-polyester composites for a prosthetic running blade. Journal of Reinforced Plastics \& Composites, vol. 40, no. 3-4, p. 118133, D0I:10.1177/0731684420949662.

[10] Fey, N.P., Klute, G.K., Neptune, R.R. (2011). The influence of energy storage and return foot stiffness on walking mechanics and muscle activity in below-knee amputees. Clinical Biomechanics, vol. 26, no. 10, p. 1025-1032, D0l:10.1016/j. clinbiomech.2011.06.007.

[11] Pérez-Carabaza, S., Scherer, J., Rinner, B., López-Orozco, J.A., Besada-Portas, E. (2019). UAV trajectory optimization for minimum time search with communication constraints and collision avoidance. Engineering Applications of Artificial Intelligence, vol. 85, p. 357-371, D0I:10.1016/j. engappai.2019.06.002.

[12] Yu, Q., Cai, M., Shi, Y., Fan, Z. (2014). Optimization of the energy efficiency of a piston compressed air engine. Strojniški vestnik - Journal of Mechanical Engineering, vol. 60, no. 6, p. 395-406, Dol:10.5545/sv-jme.2013.1383.

[13] Cheng, J., Wang, L., Xiong, Y. (2018). An improved cuckoo search algorithm and its application in vibration fault diagnosis for a hydroelectric generating unit. Engineering Optimization, vol. 50, no. 9, p. 1593-1608, D0l:10.1080/0305 215X.2017.1401067.

[14] Joly, M.M., Verstraete, T., Paniagua, G. (2013). Differential evolution based soft optimization to attenuate vane-rotor shock interaction in high-pressure turbines. Applied Soft Computing, vol. 13, no. 4, p. 1882-1891, D0l:10.1016/j. asoc.2012.12.005.

[15] Jouhaud, J.-C., Sagaut, P., Montagnac, M., Laurenceau, J. (2007). A surrogate-model based multidisciplinary shape optimization method with application to a 2D subsonic airfoil. Computers and Fluids, vol. 36, no. 3, p. 520-529, DOI:10.1016/j.compfluid.2006.04.001.

[16] White, D.A., Arrighi, W.J., Kudo, J., Watts, S.E. (2019). Multiscale topology optimization using neural network surrogate models. Computer Methods in Applied Mechanics and Engineering, vol. 346, p. 1118-1135, D0l:10.1016/j. cma.2018.09.007.

[17] Fan, X., Wang, P., Hao, F.F. (2019). Reliability-based design optimization of crane bridges using Kriging-based surrogate models. Structural and Multidisciplinary Optimization, vol. 59, p. 993-1005, DOl:10.1007/s00158-018-2183-0.

[18] Francisco, M.B, Junqueira, D.M., Oliver, G.A., Junho Pereira, J.L., da Cunha, S.S.Jr.Jr., Gomes, G.F. (2020). Design optimizations of carbon fibre reinforced polymer isogrid lower limb prosthesis using particle swarm optimization and 
Lichtenberg algorithm. Engineering Optimization, p. 1-24, DOI:10.1080/0305215X.2020.1839442.

[19] Zhai, Y., Liang, S. (2017). Optimal lay-ups to maximize loss factor of cross-ply composite plate. Composite Structures, vol. 168, p. 597-607, D0I:10.1016/j.compstruct.2017.01.019.

[20] Hobara, H., Baum, B.S., Kwon, H.-J., Linberg, A., Wolf, E.J., Miller, R.H., Shim, J.K. (2014). Amputee locomotion: Lower extremity loading using running-specific prostheses. Gait \& Posture, vol. 39, no. 1, p. 386-390, D0l:10.1016/j. gaitpost.2013.08.010.

[21] Askarzadeh, A. (2016). A novel metaheuristic method for solving constrained engineering optimization problems: Crow search algorithm. Computers \& Structures, vol. 169, p. 1-12, D0I:10.1016/j.compstruc.2016.03.001.

[22] Clayton, N., Emery, N. (2005). Corvid cognition. Current Biology, vol. 15, no. 3, p. R80-R81, D0l:10.1016/j. cub.2005.01.020.

[23] Giustolisi, O., Laucelli, D. (2005). Improving generalization of artificial neural networks in rainfall-runoff modelling. Hydrological Sciences Journal, vol. 50, no. 3, p. 457, DOI:10.1623/hysj.50.3.439.65025.

[24] Koopialipoor, M., Ghaleini, E.N., Haghighi, M., Kanagarajan, S., Maarefvand, P., Mohamad, E.T. (2019). Overbreak prediction and optimization in tunnel using neural network and bee colony techniques. Engineering with Computers, vol. 35, no. 4, p. 1191-1202, D0I:10.1007/s00366-018-0658-7.

[25] Tanzifi, M., Hosseini, S.H., Kiadehi, A.D., Olazar, M., Karimipour, K., Rezaiemehr, R., Ali, I. (2017). Artificial neural network optimization for methyl orange adsorption onto polyaniline nano-adsorbent: Kinetic, isotherm and thermodynamic studies. Journal of Molecular Liquids, vol. 244, p. 189-200, DOI:10.1016/j.molliq.2017.08.122.

[26] Afram, A., Janabi-Sharifi, F., Fung, A.S., Raahemifar, K. (2017). Artificial neural network (ANN) based model predictive control (MPC) and optimization of HVAC systems: A state of the art review and case study of a residential HVAC system. Energy and Buildings, vol. 141, p. 96-113, D0l:10.1016/j. enbuild.2017.02.012.

[27] Tsai, S.W., Wu, E.M. (1971). A general theory of strength for anisotropic materials. Journal of Composite Materials, vol. 5, no. 1, p. 58-80, D0I:10.1177/002199837100500106.
[28] Rahman, M., Bennett, T., Glisson, D., Beckley, D., Khan, J. (2014). Finite element analysis of prosthetic running blades using different composite materials to optimize performance. ASME International Mechanical Engineering Congress and Exposition, vol. 10, Dol:10.1115/IMECE2014-37293.

[29] Beck, O.N., Taboga, P., Grabowski, A.M. (2016). Characterizing the mechanical properties of running-specific prostheses. PLOS ONE, vol. 11, no. 12, p. 1-16, D0I:10.1371/journal. pone.017376.

[30] Kayri, M. (2016). Predictive abilities of Bayesian regularization and Levenberg-Marquardt algorithms in artificial neural networks: A comparative empirical study on social data. Mathematical and Computational Applications, vol. 21, no. 2, p. 1-11, D0l:10.3390/mca21020020.

[31] Blaifi, S., Moulahoum, S., Taghezouit, B., Saim A., (2019). An enhanced dynamic modeling of PV module using LevenbergMarquardt algorithm. Renewable Energy, vol. 135, p. 745-760, DOI:10.1016/j.renene.2018.12.054.

[32] Bonakdari, H., Sheikh Khozani, Z., Zaji, A.H., Asadpour, N. (2018). Evaluating the apparent shear stress in prismatic compound channels using the genetic algorithm based on multi-layer perceptron: A comparative study. Applied Mathematics and Computation, vol. 338, p. 400-411, DOI:10.1016/j.amc.2018.06.016.

[33] Kong, Y. S., Abdullah, S., Schramm, D., Omar, M. Z., Haris, S.M. (2019). Optimization of spring fatigue life prediction model for vehicle ride using hybrid multi-layer perceptron artificial neural networks. Mechanical Systems and Signal Processing, vol. 122, p. 597-621, D0I:10.1016/j.ymssp.2018.12.046.

[34] Despagne, F., Luc Massart, D. (1998). Neural networks in multivariate calibration. Analyst, vol. 123, no. 11, p. 157R-178R, D0I:10.1039/A805562I.

[35] Garson, G.D. (1991). Interpreting neural-network connection weights. Al Expert, vol. 6, 47-51.

[36] Rosel-Solis, M., Molina-Salazar, J., Dávalos-Ramírez, J.0., Pimentel-Mendoza, A.B., Vega, Y. (2019). Analysis of characteristics of composite materials built on abs cores prepared by additive manufacturing. DYNA Ingeniería e Industria, vol. 94, no. 3, p. 286-291, D0l:10.6036/8980. 